\title{
Randomized, controlled trial of TNF- $\alpha$ antagonist in CTL-mediated severe cutaneous adverse reactions
}

\author{
Chuang-Wei Wang, ${ }^{1,2}$ Lan-Yan Yang, ${ }^{3}$ Chun-Bing Chen, ${ }^{1}$ Hsin-Chun Ho, ${ }^{1,4}$ Shuen-lu Hung, ${ }^{5}$ Chih-Hsun Yang, ${ }^{1,4}$ Chee-Jen Chang, ${ }^{6,7}$ \\ Shih-Chi Su, ${ }^{1,8}$ Rosaline Chung-Yee Hui, ${ }^{1,4}$ See-Wen Chin, ${ }^{1}$ Li-Fang Huang, ${ }^{3}$ Yang Yu-Wei Lin, ${ }^{1}$ Wei-Yang Chang, ${ }^{3}$ \\ Wen-Lang Fan, ${ }^{8}$ Chin-Yi Yang, ${ }^{1}$ Ji-Chen Ho, ${ }^{4,9}$ Ya-Ching Chang, ${ }^{1,4}$ Chun-Wei Lu, ${ }^{1,4}$ Wen-Hung Chung, ${ }^{1,2,4,8}$ \\ and the Taiwan Severe Cutaneous Adverse Reaction (TSCAR) Consortium ${ }^{10}$ \\ 'Department of Dermatology, Drug Hypersensitivity Clinical and Research Center, Chang Gung Memorial Hospital (CGMH), Linkou, Taipei and Keelung, Taiwan. ${ }^{2}$ Chang Gung Immunology Consortium, \\ CGMH and Chang Gung University, Taiwan. ${ }^{3}$ Clinical Trial Center, CGMH, Linkou, Taiwan. ${ }^{4}$ College of Medicine, Chang Gung University, Taoyuan, Taiwan. ${ }^{5}$ Department and Institute of Pharmacology, School \\ of Medicine, Infection and Immunity Research Center, National Yang-Ming University, Taipei, Taiwan. ${ }^{6}$ Graduate Institute of Clinical Medical Science, Clinical Informatics and Medical Statistics Research \\ Center, Chang Gung University, Taoyuan, Taiwan. ${ }^{7}$ Biostatistical Center for Clinical Research, CGMH, Linkou, Taiwan. ${ }^{8}$ Whole-Cenome Research Core Laboratory of Human Diseases, CGMH, Keelung, Taiwan. \\ ${ }^{9}$ Department of Dermatology, Drug Hypersensitivity Clinical and Research Center, CGMH, Chiayi, Taiwan. ${ }^{10}$ The TSCAR Consortium is detailed in the Supplemental Acknowledgments.
}

BACKGROUND. Cytotoxic T lymphocyte-mediated (CTL-mediated) severe cutaneous adverse reactions (SCARs), including Stevens-Johnson syndrome (SJS) and toxic epidermal necrolysis (TEN), are rare but life-threatening adverse reactions commonly induced by drugs. Although high levels of CTL-associated cytokines, chemokines, or cytotoxic proteins, including TNF- $\alpha$ and granulysin, were observed in SJS-TEN patients in recent studies, the optimal treatment for these diseases remains controversial. We aimed to evaluate the efficacy, safety, and therapeutic mechanism of a TNF- $\alpha$ antagonist in CTL-mediated SCARs.

METHODS. We enrolled 96 patients with SJS-TEN in a randomized trial to compare the effects of the TNF- $\alpha$ antagonist etanercept versus traditional corticosteroids.

RESULTS. Etanercept improved clinical outcomes in patients with SJS-TEN. Etanercept decreased the SCORTEN-based predicted mortality rate (predicted and observed rates, $17.7 \%$ and $8.3 \%$, respectively). Compared with corticosteroids, etanercept further reduced the skin-healing time in moderate-to-severe SJS-TEN patients (median time for skin healing was 14 and 19 days for etanercept and corticosteroids, respectively; $P=0.010$ ), with a lower incidence of gastrointestinal hemorrhage in all SJS-TEN patients ( $2.6 \%$ for etanercept and $18.2 \%$ for corticosteroids; $P=0.03$ ). In the therapeutic mechanism study, etanercept decreased the TNF- $\alpha$ and granulysin secretions in blister fluids and plasma (45.7\%-62.5\% decrease after treatment; all $P<0.05$ ) and increased the Treg population ( 2 -fold percentage increase after treatment; $P=0.002$ ), which was related to mortality in severe SJS-TEN.

CONCLUSIONS. The anti-TNF- $\alpha$ biologic agent etanercept serves as an effective alternative for the treatment of CTL-mediated SCARs.

TRIAL REGISTRATION. ClinicalTrials.gov NCT01276314.

FUNDING. Ministry of Science and Technology of Taiwan.

\section{Introduction}

Stevens-Johnson syndrome (SJS) and its related disease, toxic epidermal necrolysis (TEN), are severe cutaneous adverse reac-

Conflict of interest: The authors have declared that no conflict of interest exists. Role of funding source: This work was supported by grants from the National Science Council of Taiwan (MOST101-2320-B-010-072-MY3, MOST101-2321-B-010-027, MOST101-2628-B-182-001-MY3, MOST101-2321-B-182-008, MOST102-2314-B-010014-MY3, MOST102-2321-B-182-006, and MOST103-2321-B-182-001); the CGMH (BMRPG-290011, OMRPG-2C0011, OMRPG-2C0021, CMRPG-290051-3, CMRPG-3D0351-2, CMRPG-3D0361-2, CORPG3F0041-2, and CLRPG-2E0051); and the Ministry of Health and Welfare of Taiwan (DOHW103-TDU-B-212-113003, DOHW104-TDU-B-212-113003, and DOHW105-TDU-B-212-113003).

Submitted: February 10, 2017; Accepted: December 19, 2017.

Reference information: J Clin Invest. 2018;128(3):985-996.

https://doi.org/10.1172/JC193349. tions (SCARs) involving keratinocyte apoptosis and detachment of the epidermis. Allopurinol and carbamazepine are the most common causes of SCARs in different ethnic groups, including Asians and Europeans (1-3). SJS and TEN are considered to be on the same disease spectrum, with the difference being in the extent of epidermal detachment. Patients with SJS have less than $10 \%$ body surface area (BSA) epidermal detachment, while patients with TEN have more than $30 \%$ BSA epidermal detachment. The 10\%-30\% epidermal detachment is defined as the SJS-TEN overlap $(1,4)$. Both SJS and TEN patients often have mucocutaneous eruptions, and oral mucosa is one of most commonly affected areas. Although SJS and TEN are rare (0.4 6 cases per million persons per year), they are highly lethal disorders, with mortality rates as high as approximately $5 \%$ to $12.5 \%$ for SJS and $50 \%$ for TEN $(1,5)$. 
A

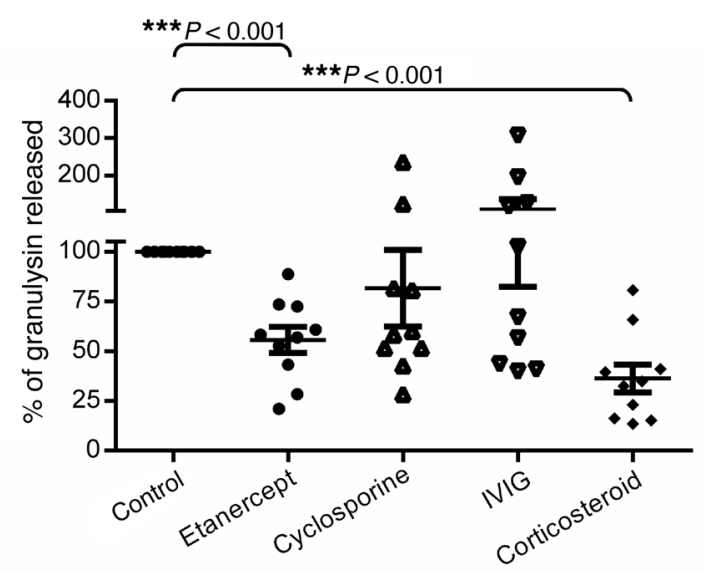

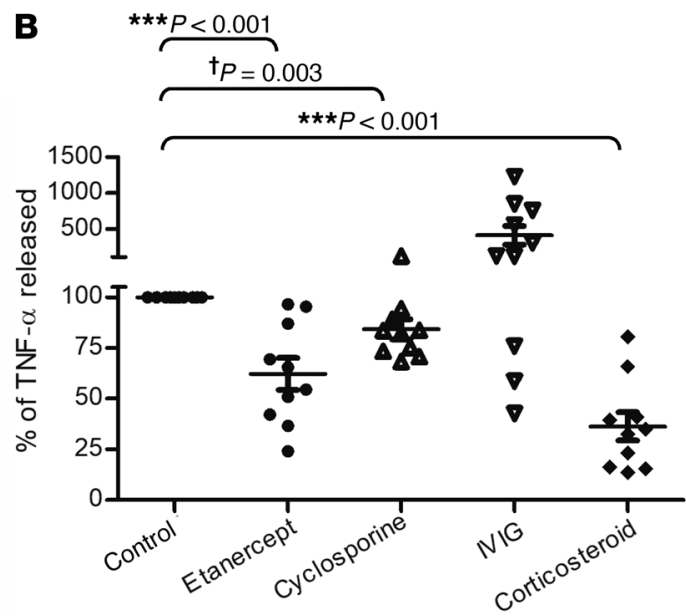

Figure 1. Preclinical testing of etanercept. Release of granulysin (A) and TNF- $\alpha$ (B) was measured by ex vivo assay in blister cells from SJS-TEN patients $(n=10)$. Blister cells were treated with DMSO (Control), $5 \mu \mathrm{g} / \mathrm{ml}$ etanercept, $400 \mathrm{ng} / \mathrm{ml}$ cyclosporine, $12 \mathrm{mg} / \mathrm{ml} \mathrm{IVIG}$, or $10 \mu \mathrm{g} / \mathrm{ml}$ corticosteroid for $48 \mathrm{hours}$. All drug concentrations were determined by clinical use. Data are presented as the mean \pm SEM and are representative of 3 independent experiments. ${ }^{* *} P<0.01$ and ${ }^{* * *} P<0.001$, by Student's $t$ test; ${ }^{\dagger} P=0.003$, by Student's $t$ test.

Over the past 2 decades, studies have shown that the pathomechanism of SJS-TEN is associated with the effector cytotoxic T lymphocytes (CTLs) that recognize culprit drugs presented by HLA class I molecules on keratinocytes (6-9). The hypothesis is not only supported by genetic studies (e.g., the strong genetic association between HLA-B ${ }^{\star}$ 15:02 and carbamazepine-SJS-TEN [ref. 6]; HLA-B ${ }^{\star}$ 58:01 and allopurinol-induced SJS-TEN [ref. $7])$, but also by functional studies $(8,10,11)$. We have previously identified secretory granulysin as a key mediator that leads to disseminated keratinocyte apoptosis and detachment of the epidermis and mucous membranes in SJS-TEN $(12,13)$. Granulysin is known to be generated by CTLs, NK cells, and NKT cells and released into the extracellular environment along with other cytotoxic mediators (such as soluble FasL, granzyme B, and perforin) $(12,14-16)$. In addition, several studies have reported that TNF- $\alpha$, a proinflammatory cytokine released by activated keratinocytes and macrophages (17), is present in the plasma and blister fluids of SJS-TEN patients $(18,19)$ and appears to serve as a potential inducer of keratinocyte apoptosis in SJS-TEN $(20,21)$.

So far, an optimal treatment guideline for CTL-mediated SCARs is still lacking. Systemic corticosteroids (22), intravenous immunoglobulin (IVIG) (23), and cyclosporine (24) are often used to treat CTL-mediated SCARs; however, the clinical outcomes of these therapies remain controversial $(25,26)$. Recently, several studies revealed that treatment with anti-TNF- $\alpha$ biologic agents appeared beneficial in patients with TEN and TEN-like acute cutaneous lupus erythematosus (17, 27-36). However, all of these studies were based on case reports and lacked a randomized, systematic trial design compared with traditional therapy studies. Here, we conducted a prospective, open-label, randomized comparison study of etanercept versus corticosteroids for the treatment of patients with SJSTEN. Etanercept (Enbrel) is a chimeric monoclonal antibody engineered by fusing the extracellular-binding domain of human TNF receptor 2 (TNFR2, also known as p75) to act as a TNF- $\alpha$ antagonist. Although the US FDA has approved the use of etanercept for treating autoimmune diseases, including rheumatoid arthritis, anky- losing spondylitis, juvenile rheumatoid arthritis, plaque psoriasis, and psoriatic arthritis, its use in treating CTL-mediated SCARs has not yet been approved (37). We aimed to evaluate the efficacy and safety of etanercept for CTL-mediated SCARs treatment in this randomized trial and further investigate the therapeutic mechanism of etanercept in CTL-mediated SCARs.

\section{Results}

Preclinical testing of etanercept: ex vivo testing for potential inhibitors in cytotoxic blister cells from patients with CTL-mediated SCARs. We previously identified granulysin as a key mediator for disseminated keratinocyte death in patients with SJS-TEN (12), and TNF- $\alpha$ has been found to act as a potential inducer of keratinocyte apoptosis in TEN $(20,21)$. To evaluate the immunosuppressive effects of etanercept (a TNF- $\alpha$ antagonist) in comparison with those of other traditional medications (such as cyclosporine, IVIG, and corticosteroid), we first performed ex vivo testing in blister cells from 10 patients with SJS-TEN enrolled in our study from 2007 to 2009. The main immunophenotypes of the cells present in the blister fluids from these patients were composed of $\mathrm{CD}^{+} \mathrm{CD}^{+} \mathrm{CTL}$ subset $(51.57 \% \pm$ $5.00 \%)$ and $\mathrm{CD}^{+} \mathrm{CD}^{+} 6^{+} \mathrm{NKT}$ cells $(20.97 \% \pm 2.73 \%)$ (Supplemental Figure 1 and Supplemental Table 1; supplemental material available online with this article; https://doi.org/10.1172/JCI93349DS1). Our results showed that treatment with etanercept for 48 hours significantly reduced the secretion of granulysin (Figure $1 \mathrm{~A} ; 44.4 \% \pm 3.1 \%$ decrease; $P<0.001$, by Student's $t$ test) and TNF- $\alpha$ (Figure 1B; $35.4 \%$ $\pm 1.6 \%$ decrease; $P<0.001$ ). Furthermore, treatment with corticosteroids showed a significant reduction in granulysin and TNF- $\alpha$ levels; however, no obvious effect on the incubation of cyclosporine or IVIG was observed (Figure 1, A and B). These results indicated that etanercept was capable of inhibiting granulysin and TNF- $\alpha$ secretion in blister cells from patients with CTL-mediated SCARs.

Clinical trial participants. For the clinical trial, we recruited 96 participants from 2009 to 2015 . A detailed study profile is shown in Figure 2. We determined whether the enrolled participants had SJS-TEN using the Registry of Severe Cutaneous Adverse Reac- 
Table 1. Demographic and baseline clinical characteristics of the trial participants

\begin{tabular}{|c|c|c|c|c|}
\hline Characteristic & $\begin{array}{c}\text { All } \\
(n=91)\end{array}$ & $\begin{array}{l}\text { Etanercept } \\
(n=48)\end{array}$ & $\begin{array}{l}\text { Corticosteroid }^{\mathrm{A}} \\
\qquad(n=43)\end{array}$ & $P$ value \\
\hline Age, years, mean $\pm S D$ & $56.09 \pm 20.81$ & $52.73 \pm 16.78$ & $59.84 \pm 24.20$ & $0.112^{\mathrm{B}}$ \\
\hline Sex, $n(\%)$ & & & & $0.658^{c}$ \\
\hline Male & $40(44.0)$ & $20(41.7)$ & $20(46.5)$ & \\
\hline Female & $51(56.0)$ & $28(58.3)$ & $23(53.5)$ & \\
\hline Skin detachment, $n$ (\%) & & & & $0.858^{c}$ \\
\hline$B S A \geq 10 \%$ & 35 (38.5) & $18(37.5)$ & $17(39.5)$ & \\
\hline$B S A<10 \%$ & $56(61.5)$ & $30(62.5)$ & $26(60.5)$ & \\
\hline $\begin{array}{l}\text { Number of sites with } \\
\text { mucous erosion, } n(\%)\end{array}$ & & & & $0.663^{\circ}$ \\
\hline One & $21(22.8)$ & $12(25.0)$ & $9(20.9)$ & \\
\hline Two & $28(30.8)$ & $16(33.3)$ & $12(27.9)$ & \\
\hline Three & $42(46.2)$ & $20(41.7)$ & $22(51.2)$ & \\
\hline Blister/erosion, $n$ (\%) & & & & $0.266^{c}$ \\
\hline- & $11(12.1)$ & $4(8.3)$ & $7(16.3)$ & \\
\hline+ & $80(87.9)$ & $44(91.7)$ & $36(83.7)$ & \\
\hline Fever $\left(>38^{\circ} \mathrm{C}\right), n(\%)$ & & & & $0.419^{\mathrm{c}}$ \\
\hline No & $34(37.4)$ & $16(33.3)$ & $18(41.9)$ & \\
\hline Yes & $57(62.6)$ & $32(66.7)$ & $25(58.1)$ & \\
\hline History of malignancy, $n$ (\%) & & & & $0.967^{c}$ \\
\hline No & $78(85.7)$ & $41(85.4)$ & $37(86.0)$ & \\
\hline Yes & $13(14.3)$ & $7(14.6)$ & $6(14.0)$ & \\
\hline Heart rate, /min, mean \pm SD & $93.41 \pm 15.68$ & $95.33 \pm 16.52$ & $91.23 \pm 13.75$ & $0.200^{\mathrm{B}}$ \\
\hline BUN, mg/dl, mean \pm SD & $26.03 \pm 29.1$ & $20.38 \pm 18.33$ & $32.33 \pm 36.57$ & $0.057^{\mathrm{B}}$ \\
\hline $\mathrm{HCO3}^{-}, \mathrm{mEq} / \mathrm{l}$, mean $\pm \mathrm{SD}$ & $22.74 \pm 4.08$ & $23.09 \pm 4.25$ & $22.34 \pm 3.84$ & $0.373^{\mathrm{B}}$ \\
\hline Clucose, $\mathrm{mg} / \mathrm{dl}$, mean \pm SD & $145.29 \pm 76.53$ & $137.6 \pm 73.59$ & $153.86 \pm 78.56$ & $0.313^{\mathrm{B}}$ \\
\hline GOT, KU/ml, mean \pm SD & $62.44 \pm 87.63$ & $56.63 \pm 72.65$ & $69.24 \pm 101.30$ & $0.508^{\mathrm{B}}$ \\
\hline $\mathrm{CPT}, \mathrm{KU} / \mathrm{ml}$, mean \pm SD & $59.38 \pm 78.78$ & $55.67 \pm 78.02$ & $63.53 \pm 79.07$ & $0.635^{\mathrm{B}}$ \\
\hline Eosinophil counts, $\%$, mean \pm SD & $2.73 \pm 3.46$ & $2.37 \pm 2.72$ & $3.16 \pm 4.11$ & $0.299^{\mathrm{B}}$ \\
\hline
\end{tabular}

${ }^{A}$ Reference group. ${ }^{B} P$ value was calculated by Student's $t$ test. ${ }^{C} P$ value was calculated by unconditional $z$-pooled test. ${ }^{D} P$ value was calculated by $\chi^{2}$ test.

participants were also registered in the RegiSCAR database and were subsequently evaluated during the biannual RegiSCAR meeting (subjects S029, S030, S032, S071, S077, etc.; Supplemental Table 4). These participants, sent for a second evaluation during the RegiSCAR meeting, were independent of this clinical trial and were all determined to have SJS-TEN. Table 1 lists the demographic and clinical characteristics of the trial participants identified as having SJS-TEN. Baseline age, sex, skin detachment, mucosal involvement, and clinical laboratory measurements were similar between the etanercept- and corticosteroidtreated groups (Table 1). A total of 71 participants completed the study: 38 and 33 participants were treated with etanercept and corticosteroids, respectively (Figure 2). Data on the participants who did not complete the study are reported in Supplemental Table 3.

Clinical improvement in CTL-mediated SCAR patients treated with etanercept. The primary monitoring parameters of the clinical endpoints were analyzed according to the time required for complete skin and oral mucosa healing and the time at which the reepithelialization of the denuded area commenced. The curves for these results are shown in Figure 3. All monitoring parameters in the total number of SJS-TEN patients did not show a significant difference between these 2 treatment groups (Figure 3, A-C). We then evaluated the efficacy of etanercept and corticosteroid treatment of SJS-TEN patients with moderate or severe skin lesions (BSA detachment $\geq 10 \%$ ), and the results are shown in Figure 3, D-F. We found that the amount of time needed for complete skin healing (Figure 3D) in patients with $10 \%$ or greater BSA detachment was significantly shorter in the etanercepttreated group than in the corticosteroid-treated group ( $P=0.010$, by Kaplan-Meier analysis). The median

tions (RegiSCAR) criteria (Supplemental Table 2) and histopathological analyses. The diagnostic evaluation was further supported by laboratory analyses of blister granulysin levels, indirect immunofluorescence (IIF), and direct immunofluorescence (DIF) to exclude other autoimmune bullous diseases. The detailed flow chart for determining whether the enrolled participants had SJSTEN is shown in Supplemental Figure 2. Among these participants, 3 failed the screening $\left(\mathrm{CD} 4\right.$ count $<200$ cells $\left./ \mathrm{mm}^{3}\right)$ and 2 had changes in their diagnosis (Figure 2 and Supplemental Table 3). A total of 91 participants were validated as SJS-TEN cases (Table 1). Seventy-eight of the ninety-one participants had skin biopsies that showed consistent pathological findings (partial epidermal necrosis, mass dyskeratosis, detached epidermis, and fullthickness epidermal necrosis) of SJS-TEN (details are provided in Supplemental Table 4 and Supplemental Figure 3). Thirteen of the enrolled participants did not undergo skin biopsies; however, they were considered probable SJS-TEN cases on the basis of the RegiSCAR criteria, with typical clinical presentations (mucosal involvement with disseminated atypical target or blistering lesions) and negative results for IIF or positive results for granulysin levels to support the diagnosis (Supplemental Table 4). Some time for skin healing in patients with $10 \%$ or greater BSA detachment was 14 days and 19 days (Figure 3D), respectively, for the etanercept and corticosteroid treatment groups. Furthermore, clinical photographs of study participants revealed that the skin lesions became drier with less rash in some patents treated with etanercept compared with the lesions of patients treated with a corticosteroid. (Figure 3G, shows 2 participants).

Assessment of mortality rates with etanercept and corticosteroid treatment. In this clinical trial, the secondary monitoring parameters were the evaluation of vital signs and adverse events. To evaluate survival rates, we first used the score of toxic epidermal necrolysis (SCORTEN), a severity-of-illness score to assess the severity and predict the mortality of SJS-TEN (38) (Supplemental Table 5). Patients in the etanercept treatment group had a mean SCORTEN of 1.85, while patients in the corticosteroid treatment group had a mean SCORTEN of 1.95 (Tables 2 and 3). We observed no statistical difference between the scores of these 2 groups $(P=0.722)$. The SCORTEN-predicted mortality rates for the etanercept and corticosteroid groups were $17.7 \%$ and $20.3 \%$, respectively (Tables 2 and 3 , and Supplemental Table 6). During the etanercept or corticosteroid treatment period, 11 patients died, 4 of whom had received 


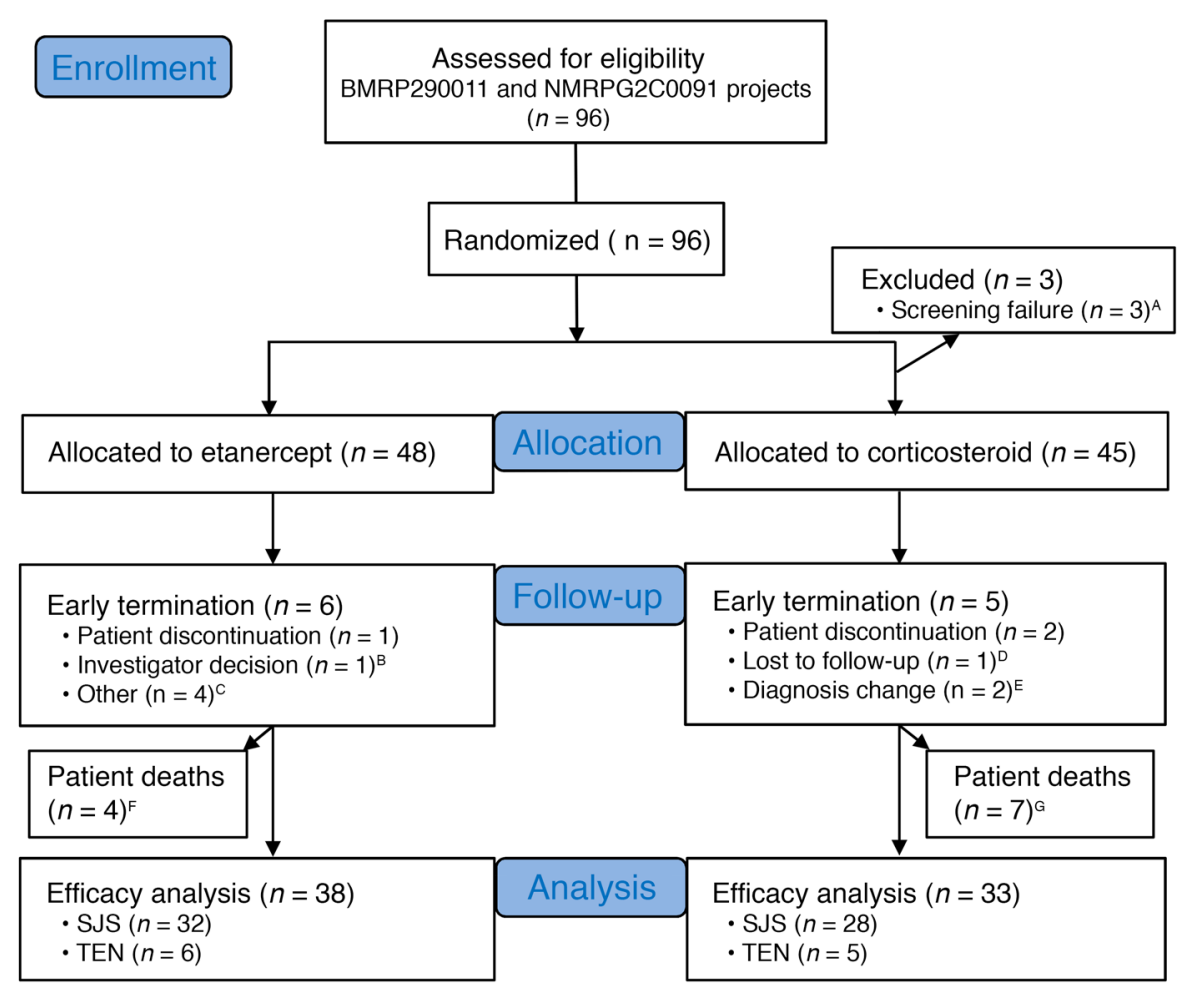

Figure 2. Flow chart for the selection of study participants. Ninety-six participants were recruited, among whom 71 completed the study. Thirty-eight patients were treated with etanercept and thirty-three patients with a corticosteroid. ${ }^{A-C}$ The group information can be found in Supplemental Table 3.

dylitis, psoriasis, and psoriatic arthritis (37). We further evaluated the incidence of adverse events and serious adverse events in this trial and determined that there were 6 serious adverse events in 5 subjects in the etanercept group and 12 serious adverse events in 9 subjects in the corticosteroid group (Table 5). These serious adverse effects included sepsis, respiratory failure, upper gastrointestinal (GI) hemorrhage (grade 3), bipolar disorder, stridor, and vocal cord palsy. Among these serious adverse events, 1 upper GI hemorrhage (grade 3) event in 1 subject (S054) was considered to be possibly related to corticosteroid use; other events were considered not to be related to etanercept or corticosteroid treatment. etanercept and 7 of whom had received corticosteroid treatment (Table 4). Although there was no statistical significance, the mortality rate for the etanercept treatment group was lower than that of the corticosteroid treatment group ( $8.3 \%$ vs. $16.3 \%, P=0.266)$ (Table 4). The causes of death for these participants are listed in Table 5 and Supplemental Table 3. The SCORTENs of the deceased participants are shown in Table 5. All 4 deceased participants in the etanercept treatment group were TEN patients with a SCORTEN of 4 . However, in the corticosteroid treatment group, 2 of the deceased patients had a SCORTEN of 3 , and 5 of the deceased participants had a SCORTEN 4 or higher.

Safety evaluation of etanercept in CTL-mediated SCAR patients. We used 25 or $50 \mathrm{mg}$ ( $>65 \mathrm{~kg}$ ) etanercept subcutaneously twice a week for SJS-TEN treatment, as this regimen is well established for the safe treatment of rheumatoid arthritis, ankylosing spon-

Table 2. SCORTEN for patients with CTL-mediated SCARs in the etanercept and corticosteroid treatment groups

\begin{tabular}{|c|c|c|c|c|}
\hline & \multicolumn{2}{|c|}{ Etanercept } & \multicolumn{2}{|c|}{ Corticosteroid } \\
\hline & $n$ & $\%$ & $n$ & $\%$ \\
\hline SCORTEN 0 & 5 & 10.4 & 3 & 7.0 \\
\hline SCORTEN 1 & 18 & 37.5 & 19 & 44.2 \\
\hline SCORTEN 2 & 11 & 22.9 & 8 & 18.6 \\
\hline SCORTEN 3 & 9 & 18.8 & 5 & 11.6 \\
\hline SCORTEN 4 & 4 & 8.3 & 6 & 14.0 \\
\hline SCORTEN 5 & 0 & 0.0 & 2 & 4.7 \\
\hline SCORTEN 6 & 1 & 2.1 & 0 & 0.0 \\
\hline Total & 48 & 100 & 43 & 100 \\
\hline
\end{tabular}
The adverse events that occurred in the treatment groups are also shown in Table 5 and included hypertension, hyperglycemia, and GI hemorrhage (grade 2). Of these adverse events, there was a significantly lower incidence of GI hemorrhage (grade 2) (Table 6) in total SJS-TEN patients treated with etanercept than in those treated with corticosteroids. The incidence rate was $2.6 \%$ in the etanercept group and $18.2 \%$ in the corticosteroid group $(P=0.030$, by unconditional $z$-pooled test) (Table 6 ). We observed no statistical difference between the 2 treatment groups in the incidence rates of other adverse events. Since this trial had a limited sample size, further study will be needed to provide a full safety assessment of etanercept use in SJS-TEN patients.

Assessment of mortality rates compared with rates in the retrospective supportive care group. We next assessed the mortality rates of the etanercept and corticosteroid groups in comparison with our retrospective database of CTL-mediated SCAR patients who received supportive care from 2001 to 2008, before the clinical trial at the same hospital system (Table 7). The analysis revealed that the mortality rate for the supportive care group was $26.3 \%$. By comparison, the mortality rate of the etanercept treatment group

\section{Table 3. SCORTEN and predicted mortality rates of patients with CTL-mediated SCARs in the etanercept and corticosteroid treatment groups}

\begin{tabular}{|l|l|l|l|}
\hline SCORTEN, mean \pm SD & $1.85 \pm 1.29$ & $1.95 \pm 1.36$ & 0.722 \\
\hline Predicted mortality, \%, mean \pm SD & $17.7 \pm 20.5$ & $20.3 \pm 25$. & 0.722 \\
\hline
\end{tabular}

$P$ value was calculated by Student's $t$ test. The predicted mortality rates were calculated on the basis of SCORTEN. 
Table 4. Observed mortality rates for patients with CTL-mediated SCARs in the etanercept and corticosteroid treatment groups

\begin{tabular}{|c|c|c|c|c|c|c|}
\hline & \multicolumn{2}{|c|}{ Etanercept } & \multicolumn{2}{|c|}{ Corticosteroid $^{A}$} & \multirow{2}{*}{$\begin{array}{c}\text { OR } \\
(95 \% \mathrm{Cl})\end{array}$} & \multirow[t]{2}{*}{$P$ value } \\
\hline & $n$ & $\%$ & $n$ & $\%$ & & \\
\hline Death & 4 & 8.3 & 7 & 16.3 & 0.47 & 0.266 \\
\hline Survival & 44 & & 36 & & $(0.13-1.72)$ & \\
\hline Total & 48 & & 43 & & & \\
\hline
\end{tabular}

${ }^{A}$ Reference group. $P$ values are based on an unconditional $z$-pooled test.

was significantly lower than that of the supportive care group $(P=$ 0.026 , by unconditional $z$-pooled test; Table 7 ). The odds ratio (OR) of death for etanercept treatment versus supportive care was 0.25 (95\% CI, 0.07-0.89). However, we detected no statistical difference between the corticosteroid treatment and supportive care groups $(P=0.280)$. We further analyzed the risk factors for death in these 3 treatment groups and found that the mortality rate for the etanercept group was still lower than that of the supportive care group in patients aged 40 years or older $(\mathrm{OR}, 0.21 ; 95 \% \mathrm{CI}, 0.06-0.75 ; P=$ 0.012) (Table 7 and Table 8). Furthermore, there was no statistical difference in the average SCORTEN or in other risk factors among the 3 treatment groups (Table 7 and Table 8).

Immunologic effects of etanercept in SJS-TEN patients. The clinical outcome of the time required for skin-healing differed significantly between the etanercept and corticosteroid treatment groups, especially for moderate-to-severe SJS-TEN patients with $10 \%$ or more BSA detachment (Figure 3D). Therefore, we focused on analyzing the immunologic effects in patients with $10 \%$ BSA or greater detachment. Plasma and blister fluid samples were collected from patients with $10 \%$ or more BSA detachment for the evaluation of granulysin and TNF- $\alpha$ expression. In blister fluids, we found that granulysin and TNF- $\alpha$ secretion decreased significantly in both treatment groups on day 2 after treatment (Figure 4, A and B). Granulysin expression levels relative to
Table 5. Summary of all serious adverse events experienced by subjects in the etanercept and corticosteroid treatment groups

$\begin{array}{ll}\text { Subject } & \text { Treatment } \\ \text { S007 } & \text { Etanercept } \\ \text { S019 } & \text { Etanercept } \\ \text { S029 } & \text { Etanercept } \\ \text { S060 } & \text { Etanercept } \\ \text { S073 } & \text { Etanercept } \\ \text { C010 } & \text { Corticosteroid } \\ \text { S004 } & \text { Corticosteroid } \\ \text { S012 } & \text { Corticosteroid } \\ \text { S030 } & \text { Corticosteroid } \\ \text { S043 } & \text { Corticosteroid } \\ \text { S054 } & \text { Corticosteroid } \\ \text { S074 } & \text { Corticosteroid } \\ \text { S075 } & \text { Corticosteroid } \\ \text { S080 } & \text { Corticosteroid }\end{array}$

Serious adverse events
Sepsis
Respiratory failure
Sepsis, respiratory failure
Bipolar disorder
Respiratory failure
Respiratory failure
Sepsis, respiratory failure
Acute respiratory failure
Sepsis, respiratory failure
Sepsis
Upper GI hemorrhage (grade 3)
Sepsis
Stridor, vocal cord palsy
Respiratory failure

$\begin{array}{cc}\text { SCORTEN } & \text { Death } \\ 4 & + \\ 4 & + \\ 4 & + \\ 0 & - \\ 4 & + \\ 5 & + \\ 4 & + \\ 3 & + \\ 4 & + \\ 3 & + \\ 1 & - \\ 4 & + \\ 2 & - \\ 4 & +\end{array}$

day-0 baseline levels were reduced by $62.5 \%(P<0.001$, by Student's $t$ test $)$ and $47.8 \%(P=0.014)$ in the etanercept and corticosteroid treatment groups, respectively (Figure $4 \mathrm{~A}$ ), whereas TNF- $\alpha$ levels were reduced by $50.0 \%(P<0.001)$ and $28.89 \%$ $(P=0.011)$ in the etanercept and corticosteroid treatment groups, respectively (Figure 4B). Furthermore, we measured the expression levels of granulysin and TNF- $\alpha$ in plasma (Figure 4, $\mathrm{C}$ and D). Plasma granulysin levels were significantly decreased from the acute stage $(35.5 \pm 5.5 \mathrm{ng} / \mathrm{ml})$ to the late stage $(15.4 \pm$ $2.9 \mathrm{ng} / \mathrm{ml}$ ) in the etanercept group (56.6\% decrease; $P=0.004)$ and were significantly decreased in the corticosteroid group (acute stage $=38.0 \pm 2.9 \mathrm{ng} / \mathrm{ml}$; late stage $=16.8 \pm 3.1 \mathrm{ng} / \mathrm{ml}$; $55.8 \%$ decrease; $P<0.001$ ) (Figure $4 \mathrm{C}$ ). Plasma TNF- $\alpha$ levels were lower at the late stage than were levels at the acute stage in both the etanercept (acute stage $=38.5 \pm 7.3 \mathrm{pg} / \mathrm{ml}$; late stage $=$ $20.9 \pm 3.0 \mathrm{pg} / \mathrm{ml} ; 45.7 \%$ decrease $P=0.037)$ and corticosteroid

Table 6. Summary of the adverse events in the etanercept and corticosteroid treatment groups

\begin{tabular}{|c|c|c|c|c|c|c|c|c|c|c|c|c|c|c|c|}
\hline \multirow[t]{3}{*}{ Characteristic } & \multicolumn{5}{|c|}{ SJS-TEN $(n=71)$} & \multicolumn{5}{|c|}{ SJS-TEN BSA $\geq 10 \%(n=22)$} & \multicolumn{5}{|c|}{ SJS-TEN BSA < $10 \%(n=49)$} \\
\hline & \multicolumn{2}{|c|}{$\begin{array}{c}\text { Etanercept } \\
(n=38)\end{array}$} & \multicolumn{2}{|c|}{$\begin{array}{c}\text { Corticosteroid } \\
(n=33)\end{array}$} & \multirow[b]{2}{*}{$P$ value } & \multicolumn{2}{|c|}{$\begin{array}{c}\text { Etanercept } \\
\quad(n=11)\end{array}$} & \multicolumn{2}{|c|}{$\begin{array}{c}\text { Corticosteroid } \\
\quad(n=11)\end{array}$} & \multirow[b]{2}{*}{$P$ value } & \multicolumn{2}{|c|}{$\begin{array}{c}\text { Etanercept } \\
(n=27)\end{array}$} & \multicolumn{2}{|c|}{$\begin{array}{c}\text { Corticosteroid } \\
\quad(n=22)\end{array}$} & \multirow[b]{2}{*}{$P$ value } \\
\hline & $n$ & $\%$ & $n$ & $\%$ & & $n$ & $\%$ & $n$ & $\%$ & & $n$ & $\%$ & $n$ & $\%$ & \\
\hline+ & 6 & 15.8 & 5 & 15.2 & & 1 & 9.1 & 2 & 18.2 & & 5 & 18.5 & 3 & 13.6 & \\
\hline- & 32 & 84.2 & 28 & 84.8 & & 10 & 90.9 & 9 & 81.8 & & 22 & 81.5 & 19 & 86.4 & \\
\hline Hyperglycemia & & & & & 0.767 & & & & & 0.682 & & & & & 0.500 \\
\hline- & 30 & 78.9 & 25 & 75.8 & & 8 & 72.7 & 9 & 81.8 & & 22 & 81.5 & 16 & 72.7 & \\
\hline GI hemorrhage (grade 2) & & & & & $0.030^{A}$ & & & & & 0.343 & & & & & $0.044^{A}$ \\
\hline+ & 1 & 2.6 & 6 & 18.2 & & 1 & 9.1 & 3 & 27.3 & & 0 & 0 & 3 & 13.6 & \\
\hline - & 37 & 97.4 & 27 & 81.8 & & 10 & 90.9 & 8 & 72.7 & & 27 & 100 & 19 & 86.4 & \\
\hline
\end{tabular}

Hypertension is defined as a systolic blood pressure greater than $140 \mathrm{mmHg}$ and a diastolic blood pressure greater than $90 \mathrm{mmHg}$; hyperglycemia is defined as a blood glucose level above $200 \mathrm{mg} / \mathrm{dl}$ after a meal. All adverse events were reported during treatment. ${ }^{A}$ Statistically significant $P$ values. All $P$ values were determined using an unconditional $z$-pooled test for each category. 
Table 7. Mortality rates of patients treated with etanercept or corticosteroids compared with retrospective data on CTL-mediated SCAR patients treated with supportive care

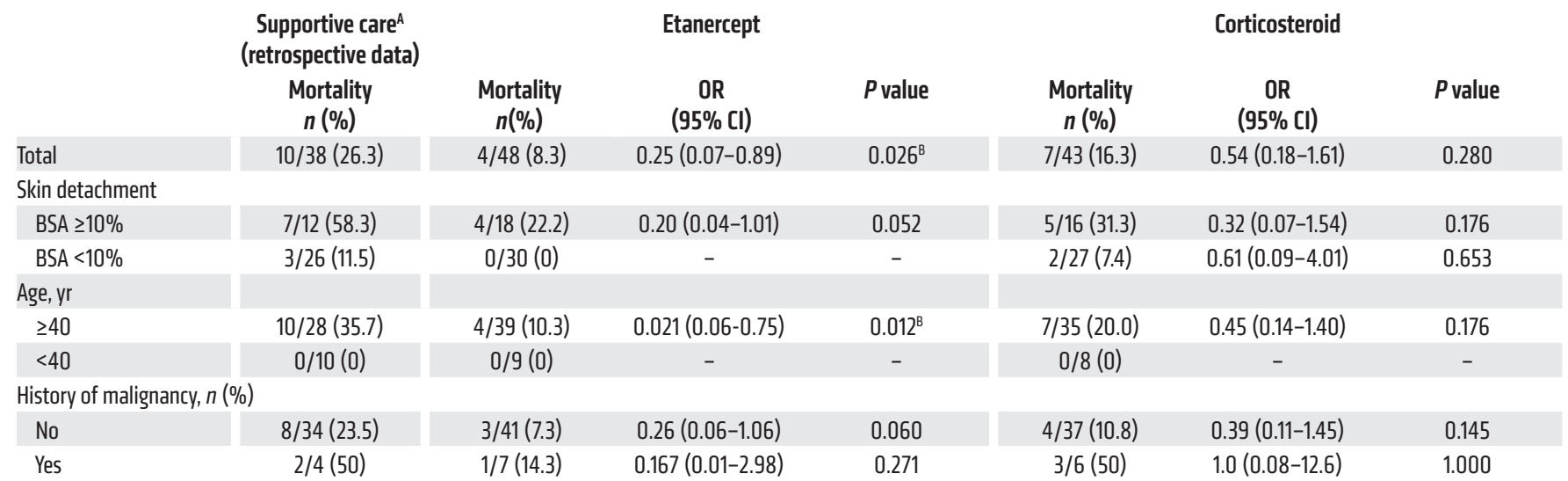

${ }^{A}$ Reference group. ${ }^{B}$ Statistically significant $P$ values. All $P$ values were calculated using an unconditional $z$-pooled test for etanercept versus supportive care and corticosteroids versus supportive care.

groups (acute stage $=32.6 \pm 2.6 \mathrm{pg} / \mathrm{ml}$; late stage $=24.3 \pm 2.3 \mathrm{pg}$ / $\mathrm{ml}$; 25.5\% decrease; $P=0.028$ ) (Figure $4 \mathrm{D}$ ).

We then measured the effects of $\mathrm{CD} 4{ }^{+} \mathrm{CD} 25^{\mathrm{hi}} \mathrm{FOXP} 3^{+}$Tregs in patients with BSA detachment of $10 \%$ or more in both treatment groups (Figure 4, E and F). The results revealed that etanercept significantly increased the Treg population, which showed mean percentages of $2.7 \% \pm 0.5 \%$ and $4.8 \% \pm 0.8 \%$ at the acute and maximum stages, respectively $(P=0.005)$ and $5.4 \% \pm 0.8 \%$ at the late stage ( $P=0.002$ vs. the acute stage) (Figure $4 \mathrm{~F})$. In contrast, we observed no significant difference in the corticosteroid group (acute, maximum, and late stages, $2.4 \% \pm 0.4 \%, 2.8 \% \pm 0.8 \%$, and $3.3 \% \pm 0.7 \%$, respectively; all $P>0.05$ vs. the acute stage). We also detected an increase in the Treg population at the recovery stage in the etanercept group of total SJS-TEN patients (acute and late stages, $2.5 \pm 0.2$ and $3.8 \pm 0.4 \%$, respectively; $P=0.035$; Supplemental Figure 4A). However, we did not observe this increase in patients with mild SJS-TEN who had less than $10 \%$ BSA detachment (Supplemental Figure 4B) or in any patients in the corticosteroid treatment group (Supplemental Figure 4).

We further examined the correlation between clinical severity and Treg populations in SJS-TEN patients enrolled in the trial and found that the Treg population at the maximum stage (average of
$8.8 \pm 5.1$ days from onset) was significantly lower in the deceased patients than in patients who survived (all deceased patients, $1.7 \%$ $\pm 0.3 \%$; all surviving patients, $3.3 \% \pm 0.3 \% ; P=0.036$ ) (Supplemental Figure 5A). We detected no significant difference in Treg populations during the acute stage in patients with SJS-TEN of different severities (Supplemental Figure 5B). We found that the Treg populations were significantly increased during the maximum stage in patients with $10 \%$ or greater BSA detachment in the etanercept treatment group compared with that in the corticosteroid treatment group ( $P=0.029$; Supplemental Figure $5 B)$. However, the Treg population was still lower during the maximum stage in the deceased patients than it was in all surviving patients with SJSTEN and BSA detachment of $10 \%$ or greater (both $P<0.05$; Supplemental Figure 5B) after etanercept treatment. Likewise, there was no significant difference in the corticosteroid group (Supplemental Figure 5B).

\section{Discussion}

This study was designed to evaluate the efficacy of etanercept (an anti-TNF- $\alpha$ biologic agent) compared with that of traditional therapy in CTL-mediated SCAR patients. Previously, several case reports demonstrated that TEN and TEN-like acute cutaneous

Table 8. Clinical characteristics as additional risk factors for mortality among patients treated with etanercept or corticosteroids compared with retrospective data on CTL-mediated SCAR patients treated with supportive care

\section{Supportive care ${ }^{A}$ (retrospective data)}

Age, yr, mean \pm SD Heart rate, /min, mean \pm SD BUN, $m g / d l$, mean \pm SD $\mathrm{HCO3}^{-}, \mathrm{mEq} / \mathrm{l}$, mean $\pm \mathrm{SD}$ Clucose, $\mathrm{mg} / \mathrm{dl}$, mean $\pm \mathrm{SD}$ SCORTEN, mean \pm SD

$56.00 \pm 24.65$
$96.81 \pm 11.99$
$24.75 \pm 16.31$
$21.38 \pm 4.11$
$157.06 \pm 59.77$
$1.97 \pm 1.29$

Etanercept

$52.73 \pm 16.78, P=0.444$
$95.33 \pm 16.52, P=0.644$
$20.38 \pm 18.33, P=0.285$
$23.09 \pm 4.25, P=0.082$
$137.6 \pm 73.59, P=0.297$
$1.85 \pm 1.29, P=0.100$
Corticosteroid

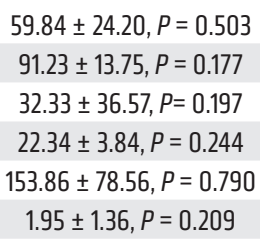

${ }^{A}$ Reference group. All $P$ values were calculated by Student's $t$ test. 
A

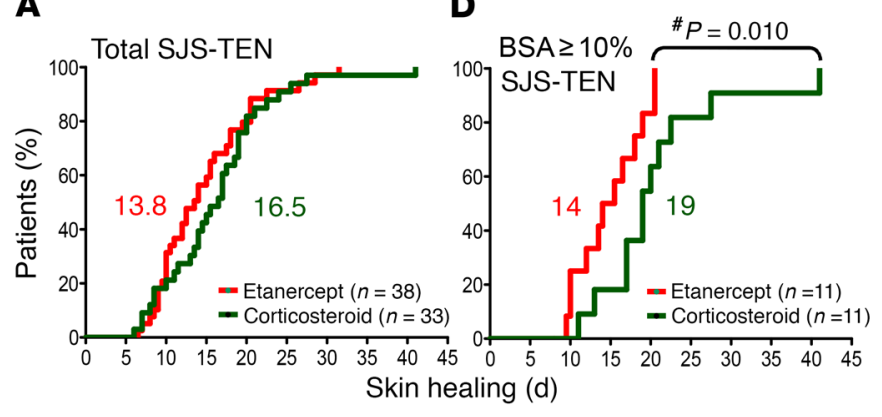

B

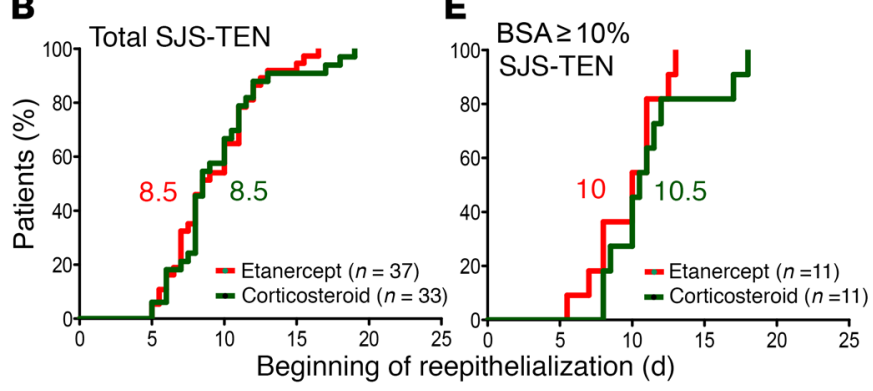

C

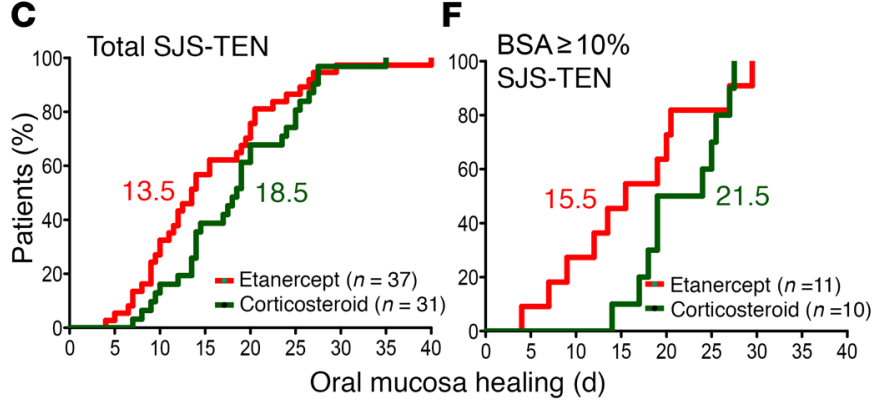

lupus erythematosus could successfully be treated with etanercept (27-32) and other TNF- $\alpha$ antagonists (17, 33-36). Paradisi et al. even observed successful treatment in 10 patients with TEN (31). However, to our knowledge, no randomized, systematic comparison of anti-TNF- $\alpha$ biologic agents versus traditional therapy has been made, nor has a mechanistic study on anti-TNF- $\alpha$ biologic agents in SJS-TEN patients been reported. In a randomized, controlled study of SJS-TEN, Pierre et al. compared thalidomide versus placebo in 22 patients with TEN (39). Our study not only enrolled the largest series of CTL-mediated SCARs patients treated with a biologic agent, but, to our knowledge, is also the first randomized, controlled trial involving the TNF- $\alpha$ antagonist in CTL-mediated SCARs.

The incidence of SJS-TEN is low, with an estimated 0.4-6 cases per million persons per year $(1,5)$; therefore, the sample size of this study is limited. Despite the sample size limitation, we ultimately enrolled 91 participants with SJS-TEN in the clinical trial. According to our retrospective database (OR, 0.25; 95\% CI, 0.07-0.89; $P=0.026$ ), the mortality rate after etanercept treatment $(8.3 \%)$ was lower than the rate predicted on the basis of patients' SCORTENs (17.7\%) and was significantly lower than that of the supportive care treatment group $(26.3 \%)$. Furthermore, the mortality rate for corticosteroid treatment (16.3\%) was also lower than the predicted rate (20.3\%), but no statistical difference was observed in the corticosteroid group compared with the etanercept and supportive care groups. Although the result
G

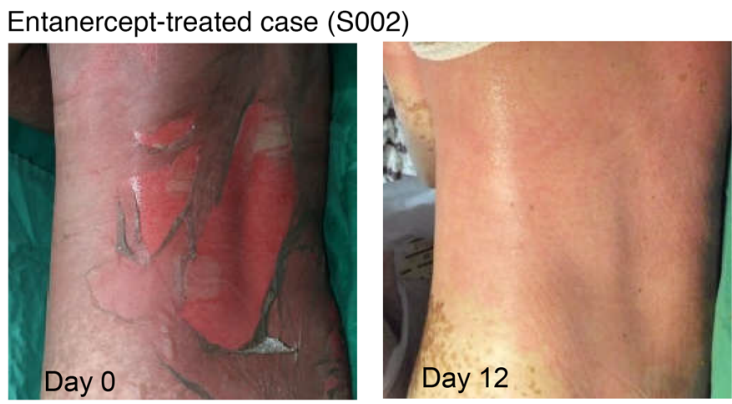

Corticosteroid-treated case (S015)

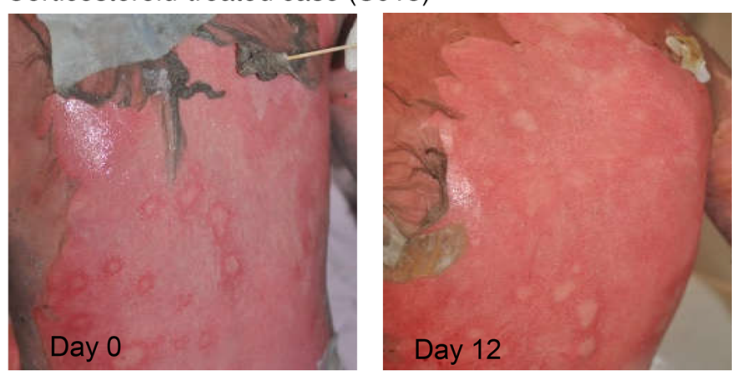

Figure 3. Clinical improvement in CTL-mediated SCAR patients after etanercept or corticosteroid treatment. Kaplan-Meier curves are shown for the time required for skin healing ( $\mathbf{A}$ and $\mathbf{D})$, beginning of reepithelialization (B and $\mathbf{E}$ ), and oral mucosa healing ( $\mathbf{C}$ and $\mathbf{F}$ ) in CTL-mediated SCAR patients in the 2 treatment groups. The numbers in red and green represent the median number of days for the etanercept and corticosteroid groups, respectively. $P$ values were calculated by the Kaplan-Meier product-limit estimates method. ${ }^{*} P=0.010$, by Kaplan-Meier product-limit estimates method. (C) Shown are representative clinical photographs of patients with TEN who received etanercept or corticosteroids.

was not significant, the corticosteroid-treated group (16.3\%) had lower mortality rates than did the supportive care group (26.3\%), which was similar to the results of a previous report (25). A larger sample size is needed to confirm the survival benefit of etanercept treatment for SJS-TEN compared with that provided by systemic corticosteroids. In addition, the causes of death in the etanercept treatment group included sepsis, serious infection, and respiratory failure, which are common causes of death in SJS-TEN patients (40). All 4 deceased participants in the etanercept group were TEN patients with a SCORTEN of 4, suggesting that the deaths of these 4 patients were related to the severity of their illness.

In our evaluation of clinical manifestations, we found that etanercept could effectively improve skin and oral mucosa healing, as well as facilitate reepithelialization. In comparison with corticosteroids, etanercept further reduced the time required for complete skin healing $(P=0.010)$ in SJS-TEN patients with moderate or severe skin involvement (BSA detachment $\geq 10 \%$ ). Corticosteroids can nonspecifically influence steroid receptormediated transcription factors that are involved in a wide variety of physiological functions, including stress response, immune reaction, carbohydrate metabolism, and protein catabolism (41). Systemic steroids have been reported to impair wound healing (42). Because etanercept specifically targets TNF- $\alpha$, it would be reasonable to expect a better skin-healing effect with etanercept than with corticosteroids. 
A

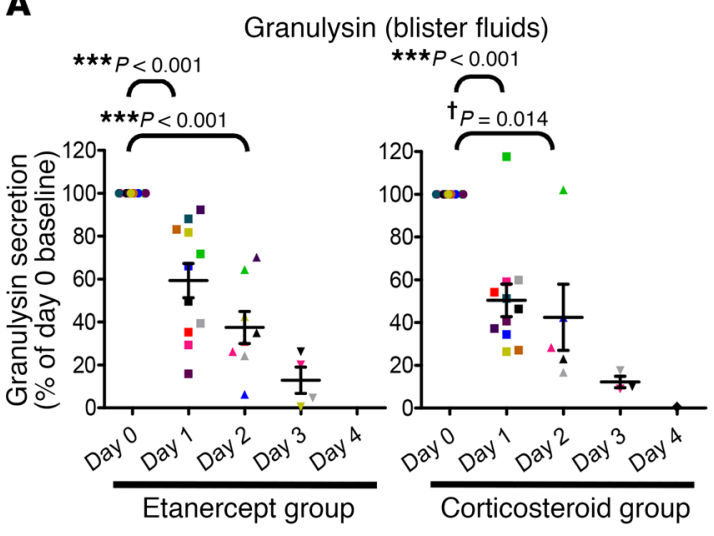

C

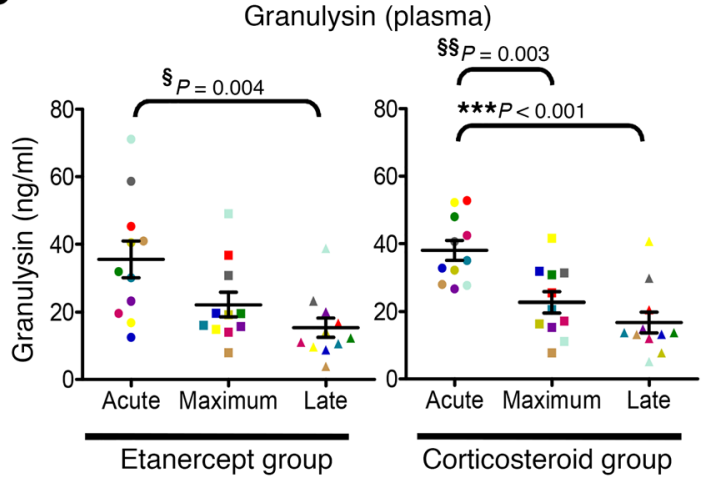

E

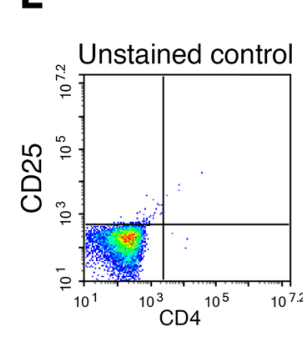

Case 5071 (acute)
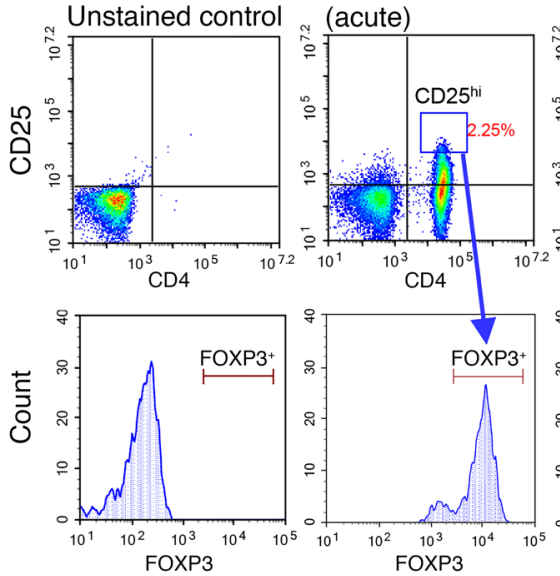

Etanercept-treated (late)

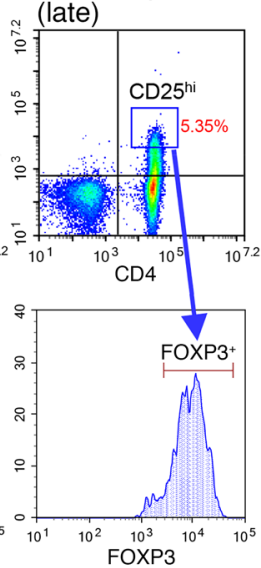

B

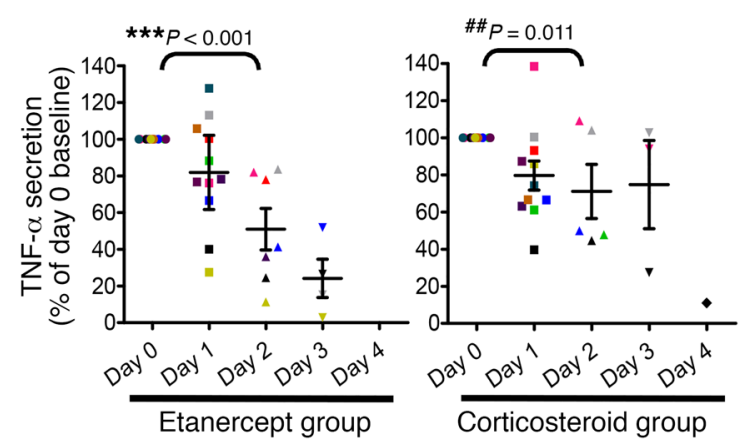

D

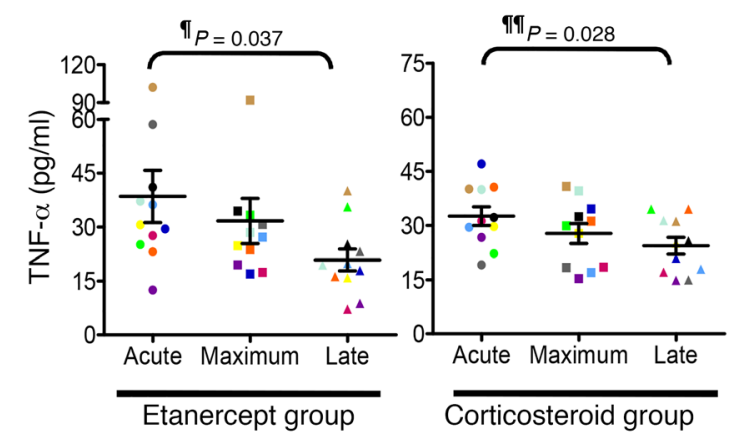

$\mathbf{F}$

TNF- $\alpha$ (blister fluids)

TNF- $\alpha$ (blister fluids)

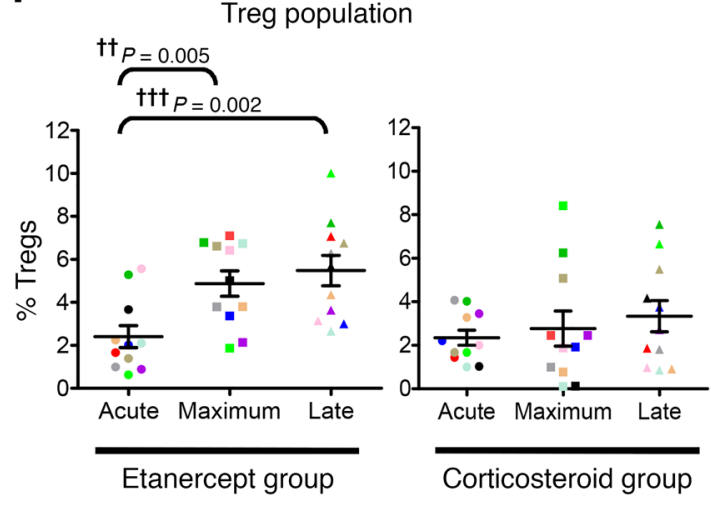

Figure 4. Immunological effects in SJS-TEN patients with $10 \%$ or greater BSA detachment after etanercept or corticosteroid treatment. Release of granulysin (A) and TNF- $\alpha$ (B) was measured in blister fluids from SJS-TEN patients with $10 \%$ or greater BSA detachment in the etanercept $(n=11)$ and corticosteroid ( $n=11)$ treatment groups. The blister fluids were continually collected on day 0 (before treatment) and each day afterward (days $1,2,3$, and 4 after treatment) until blister production stopped. Expression levels of granulysin (C) and TNF- $\alpha$ (D) in plasma were also detected. The acute stage was defined as occurring within 6 days from the onset of illness; the maximum stage was defined as the time from illness onset to maximal skin detachment;

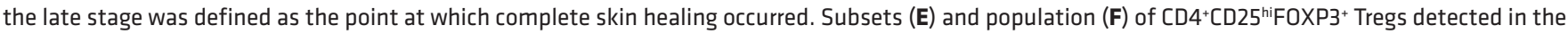
patients' blood. Data are presented as the mean \pm SEM based on $3(\mathbf{A}-\mathbf{D})$ or $2(\mathbf{F})$ independent experiments; all $P$ value was calculated by Student's $t$ test.

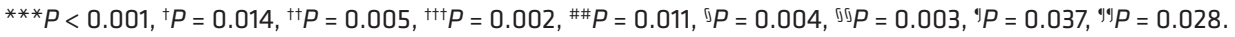

Etanercept has been approved by the US FDA to treat rheumatoid arthritis, ankylosing spondylitis, plaque psoriasis, psoriatic arthritis, and juvenile rheumatoid arthritis (37). In a long-term study, clinical safety results were reported for 2,054 patients from North America and Europe $(37,43)$. In this trial, 6 serious adverse events including sepsis and respiratory failure in
5 patients in the etanercept group were reported. We speculate that these serious adverse effects could have been related to the patients' illness. We also evaluated adverse effects in the etanercept treatment group and found a significantly lower incidence of GI hemorrhage (grade 2) $(P=0.030)$ in total SJS-TEN patients in the etanercept treatment group than in patients in the cortico- 
steroid treatment group. GI hemorrhage is a rare side effect of etanercept treatment (37) and was not found in our retrospective supportive care group (data not shown). However, systemic corticosteroids have been known to be associated with an increased risk of GI hemorrhage $(37,44)$.

TNF- $\alpha$ expression has been found to be high in plasma and blister fluids of SJS-TEN patients $(18,19)$, and it appears to be associated with the pathogenesis of CTL-mediated SCARs (21). TNF- $\alpha$ serves as a proinflammatory cytokine and is thought to play an essential role in inducing immune responses. In addition, we previously revealed that granulysin is the key mediator for keratinocyte detachment of the epidermis in SJS-TEN (12), and Kida et al. found that TNF- $\alpha$ could augment the granulysin promoter activity to regulate granulysin gene expression (45). TNF- $\alpha$ appears to be the upstream regulator of granulysin. Our results showed that secretion of TNF- $\alpha$ and granulysin in plasma and blister fluids of SJSTEN patients clearly decreased after etanercept or corticosteroid treatment. We hypothesize that etanercept attenuates granulysin expression via blockade of the TNF- $\alpha$-associated pathway.

It has been determined that Tregs are responsible for suppressing $\mathrm{T}$ cell responses. Impaired Tregs or the function of these impaired cells have been found to be associated with many autoimmune diseases as well as with SJS-TEN (46-48). Tregs expressing the FOXP3 transcription factor regulate immune suppression and induce tolerance to limit hypersensitivity and autoimmunity. In a study on SJS-TEN, Tregs isolated from patients with acute-stage TEN were found to be profoundly impaired in their suppressive function, which may be related to severe epidermal damage (49). Another recent study also revealed that the drug-specific Tregs proliferated during the recovery stage in 1 SCAR patient (50). Furthermore, several studies indicated that the expression levels of FOXP3 and the suppressive functions of Tregs were increased by anti-TNF- $\alpha$ treatment $(51,52)$. Two studies reported an increase in the Treg population in rheumatoid arthritis patients following treatment with an anti-TNF- $\alpha$ agent $(51,53)$.

In this study, we found that etanercept significantly increased Treg proportions in patients with SJS-TEN during the recovery stage, especially in those with $10 \%$ or more BSA detachment, compared with Treg proportions during the acute stage. However, no significant difference was observed after treatment with corticosteroids, which may have been due to their pan-immunosuppressive effects.

We further found that Treg populations were significantly lower in all deceased patients than in all surviving patients, suggesting that Tregs were involved in the mortality of SJS-TEN. In addition, an increase in the Treg population was detected in all surviving patients with SJS-TEN after etanercept treatment, but not in the 4 deceased patients with TEN who had received etanercept treatment. Etanercept can increase Treg populations in most SJS-TEN patients, but it may not be sufficient to restore the Treg population in patients with severe TEN because of their poor condition or other unfavorable prognostic factors such as infection. Therefore, this subset of patients may require an enhancement of the etanercept dosage. However, the therapeutic effect and optimal use of etanercept for patients with severe, rapidly progressing SJS-TEN need further exploration. Over- all, etanercept increased the proportion of Tregs and showed potential immunosuppressive and immunomodulatory effects in patients with CTL-mediated SCARs.

In conclusion, the anti-TNF- $\alpha$ biologic agent etanercept is an effective alternative treatment for CTL-mediated SCARs. In comparison with conventional systemic corticosteroids, etanercept showed a shorter skin-healing time for patients with moderateto-severe CTL-mediated SCARs, with a lower incidence of GI side effects. Our mechanism-of-action study revealed that etanercept treatment decreased the levels of granulysin and TNF- $\alpha$ in the plasma and blister fluids and increased Treg populations in patients with moderate-to-severe CTL-mediated SCARs. This study may provide a rationale for the clinical application of antiTNF- $\alpha$ biologic agents in the treatment of patients with CTLmediated SCARs.

\section{Methods}

Patient enrollment in the clinical trial. SJS-TEN patients were enrolled in the clinical trial from 2009 to 2015 at CGMH, which is the largest medical health system in Taiwan and receives SJS-TEN referral cases from other hospitals in northern Taiwan. Patients older than 4 years were eligible if they were diagnosed with SJS or TEN, characterized by rapidly developing blistering exanthema with purpuric macules, accompanied by mucosal involvement and skin detachment. A diagnosis of SJS-TEN was also made according to the phenotypic criteria of the RegiSCAR study (54-56), which are shown in Supplemental Table 2. Skin biopsies were histopathologically examined for the majority of enrolled participants (Supplemental Table 4). Only participants with probable or definite cases of SJS-TEN were enrolled in this study. The diagnostic evaluation was further supported by laboratory assessments of skin biopsies with DIF and IIF using anti-intercellular substance and anti-basement membrane zone autoantibodies (57), as well as detection of blister granulysin levels to exclude other autoimmune bullous diseases (Supplemental Figure 2). A granulysin level above $600 \mathrm{ng} / \mathrm{ml}$ (12) was considered positive for SJS-TEN (Supplemental Table 4).

In addition, to assess the patients' physical condition and vital signs, blood samples were evaluated for hematologic and biochemical values, arterial blood gas levels, purified protein derivative (PPD) levels, and viral or bacterial infection. The patients also underwent urinalysis, a urine pregnancy test, and chest x-ray. All patients were assessed and diagnosed by at least 2 experienced dermatologists.

Exclusion criteria. The following patients were excluded from the clinical trial: (a) pregnant or breastfeeding women; (b) patients with a previous allergy to any anti-TNF- $\alpha$ biological product; (c) patients with active or latent tuberculosis confirmed by chest $x$-ray; (d) patients with severe, active infection and septicemia; (e) carriers of active hepatitis B or C; (f) suspected carriers of HIV with a $\mathrm{CD} 4^{+} \mathrm{T}$ cell count below 200; and (g) patients with poor compliance or safety concerns, as judged by an investigator.

Study procedures. This was an open-label, randomized, controlled, unblinded study. Because CTL-mediated SCARs such as SJS-TEN are rare diseases, with an incidence of fewer than 10 cases per million persons per year worldwide $(1,5)$, the sample size of the trial was determined by physician estimation of the likely number of available SJS-TEN patients. We ultimately recruited 96 patients for this trial. Of these 96 patients, the first 10 were enrolled in the BMRP290011 project from 2009 to 2010, and the remaining 86 patients were enrolled 
in the NMRPG2C0091 project from 2010 to 2015. Both BMRP290011 and NMRPG2C0091 projects have the same trial design and outcome predictions as well as the same IRB numbers, but have different informed consent provisions. Given the study design and outcome predictions, we incorporated these 2 projects into 1 single trial analysis. In addition, we used different random allocation numbers for these 2 projects. Both random number lists were generated by the consulting biostatistician using SAS 9.2 and were assigned chronologically to each participant.

When a SJS-TEN patient was admitted to the hospital, a random number was generated by the clinical trial center within 1 to 2 days of the patient's initial clinical assessment, after which the investigator was given the random number and initiated the appropriate treatment. Participants with SJS-TEN were consecutively enrolled and randomly assigned at a 1:1 ratio to receive either $25 \mathrm{mg}$ (or $50 \mathrm{mg}$ ) etanercept by subcutaneous injection twice a week or $1-1.5 \mathrm{mg} / \mathrm{kg} /$ day prednisolone (systemic corticosteroid) (58) by intravenous injection until their skin lesions were healed. Since body weight could influence the drug's efficacy, $50 \mathrm{mg}$ etanercept was administered to patients who weighed more than $65 \mathrm{~kg}$. Because the injection methods are quite different for etanercept and corticosteroids, this was not a blinded clinical trial. If participants met the exclusion criteria or could not complete the study, we allowed them to undergo traditional treatment (mainly with corticosteroids or supportive care only).

In addition, we also enrolled patients from the Drug Reaction with Eosinophilia and Systemic Symptoms (DRESS) trial (ClinicalTrials.gov Identifier: NCT01276314); however, the present study only focused on the SJS-TEN patient group.

Assessment of clinical outcomes and adverse events. The main endpoint and primary monitoring parameters of this clinical trial were analyzed according to the healing of skin or mucosal lesions. Healing was defined as complete reepithelialization (i.e., the complete absence of erosions). We recorded the length of time required for the skin and oral mucosa to heal and the time required to begin reepithelialization on the denuded areas. All enrolled participates were in-patients and were monitored at least every 2 days. In addition, the secondary monitoring parameters were to evaluate the patients' adverse events and vital signs. The endpoints of secondary monitoring parameters were assessed for 3 weeks after discharge. Clinical laboratory measurements and blood and urine analyses were also performed throughout this study. At least 2 dermatologists assessed each clinical monitoring parameter and each adverse event attributable to the severity of the illness or to the drug.

Comparison of mortality rates in the different treatment groups. The clinical severity of SJS-TEN was evaluated using SCORTEN (38) (Supplemental Table 5). Using the probability of death linked to each SCORTEN, we calculated the predicted mortality in our patients. We first evaluated the mortality rates for the etanercept and corticosteroid treatment groups in this clinical trial. Because SJS-TEN is a rare and life-threatening clinical condition, patients with SJS-TEN could not be allocated to supportive care-only groups for ethical reasons in this intention-to-treat-based clinical trial. We collected retrospective data on patients with SJS-TEN who were managed by supportive care without specific treatment from our SJS-TEN registry database from 2001 to 2008 at the same CGMH health system hospital. All retrospective cases of SJS-TEN were accessed with complete, accessible electronic medical records. The mortality rate of patients in the retrospective supportive care group was compared with the results of the etanercept and corticosteroid treatment groups in this clinical trial.

Ex vivo evaluation of the anti-TNF- $\alpha$ agent and other immunosuppressants for CTL-mediated SCARs. For preclinical, ex vivo testing of potential inhibitors, SJS-TEN patients with blisters were enrolled from 2007 to 2009 at the same CGMH. The blister cells were collected before treatment. In addition, the cellular phenotypes of the blister cells from these SJS-TEN patients were characterized by different human cell markers using flow cytometry. The cell viability of the blister cells was also determined by propidium iodide staining (see the Supplemental Methods for further details).

Blister cells were isolated by centrifugation at $1,000 \mathrm{~g}$ for $5 \mathrm{~min}$ utes. A total of $1 \times 10^{5}$ cells were treated for 48 hours with potential inhibitors, including etanercept, cyclosporine, IVIG, and corticosteroids, after which culture supernatants were collected for ELISA measurement of granulysin and TNF- $\alpha$ levels. All drug concentrations are shown in Figure 1 and were determined by clinical use.

Measurement of granulysin and TNF- $\alpha$ secretion. Plasma samples from patients in acute, maximum, or late stages of SJS-TEN were obtained for both treatment groups and frozen at $-80^{\circ} \mathrm{C}$. The point of illness onset for each individual patient was also evaluated. The acute stage was defined as occurring within 6 days of the onset of illness; the maximum stage was defined as the time from illness onset to maximal skin detachment (without progression of skin detachment); and the late stage was defined as the point at which complete skin healing occurred. The average times (mean \pm SD) to reach the acute, maximum, and late stages were $4.3 \pm 1.7,9.1 \pm 4.7$, and $14.3 \pm$ 6.2 days, respectively, for the etanercept group and $5.1 \pm 2.5,8.4 \pm$ 5.8 , and $16.0 \pm 7.2$ days, respectively, for the corticosteroid group. If patients had blistering skin, the blister fluids were continually collected on day 0 (before treatment) and each day afterward (days $1,2,3$, and 4 after treatment) until blister production stopped. All assays were performed at the end of the clinical trial. To determine granulysin expression levels, the mAbs RB1 (catalog D184-3) and biotin-labeled RC8 (catalog D185-6) (MBL) were used in an ELISA, as described previously (12). The assay sensitivity for granulysin was $20 \mathrm{pg} / \mathrm{ml}$. Secreted TNF- $\alpha$ was detected using a human TNF- $\alpha$ ELISA Kit (catalog DY210-05; R\&D Systems). The assay sensitivity for TNF- $\alpha$ was $15 \mathrm{pg} / \mathrm{ml}$.

Flow cytometric analysis of Treg populations. Peripheral blood mononuclear cells (PBMCs) $\left(1 \times 10^{7}\right)$ isolated from SJS-TEN patients' blood at acute, maximum, and late stages were obtained from patients in both treatment groups and frozen at $-196^{\circ} \mathrm{C}$ in liquid nitrogen. This assay was also performed at the end of the clinical trial. To identify Treg populations, patients' PBMCs $\left(4 \times 10^{5}\right)$ were incubated with PE-labeled anti-human CD25 (catalog 555432; BD Biosciences), PerCP-labeled anti-human CD4 (catalog IM2636U; Beckman Coulter), and APC-Cy7-labeled anti-human CD45 (catalog A7117; Beckman Coulter) at $4^{\circ} \mathrm{C}$ for 30 minutes. After surface staining, the samples were fixed and permeabilized according to the instructions for the BD Fixation/Permeabilization Kit (catalog 554714; BD Bioscience). Next, the cells were stained with FITC-labeled anti-human FOXP3 (catalog 00-5523-00; Thermo Fisher Scientific). After washing, the stained cells were processed through a NovoCyte flow cytometer (ACEA Bioscience), and data were analyzed using NovoExpress 1.1.0 software (ACEA Bioscience). CD $4{ }^{+} \mathrm{CD} 25^{\text {hi }} \mathrm{FOXP}^{+} \mathrm{T}$ cells were defined as Tregs. 
Statistics. Different statistical methods were used to analyze the results. For evaluation of the primary endpoint, which was the time required to heal skin erosions and oral mucosa and to begin reepithelialization, the Kaplan-Meier product limit estimates method was performed using GraphPad Prism 5 (GraphPad Software). To determine the categorical variables within the small samples on a contingency table, an unconditional $z$-pooled test was performed (59). The secondary monitoring parameters (e.g., adverse events and mortality rate) and several baseline clinical characteristics (e.g., sex, skin detachment, blister/erosion, fever, and history of malignancy) of the 2-group comparisons were analyzed using an unconditional $z$-pooled test. The predicted mortality rates were calculated using the SCORTEN scale (Supplemental Tables 5 and 6). A $\chi^{2}$ test was used for 3-group comparisons of baseline characteristic mucosal involvement. Furthermore, statistical comparisons between 2 variables were performed by 2-tailed Student's $t$ test using GraphPad Prism 5 (GraphPad Software). A 2-tailed Student's $t$ test was used for analysis of SCORTEN and some of the baseline clinical characteristics (including age, heart rate, blood urea nitrogen [BUN], bicarbonate $\left[\mathrm{HCO}^{-}\right]$, glucose, glutamate oxaloacetate transaminase [GOT], glutamic pyruvic transaminase [GPT], and eosinophil counts) as well as immunologic effects (such as granulysin and TNF- $\alpha$ secretion and Treg populations) between 2 treatment groups. Analysis of the immunologic effects was performed at the end of the clinical trial, and the data are presented as the mean \pm SEM. Differences were considered statistically significant at a $P$ value of less than 0.05.

Study approval. Each study participant or the closest relative provided written informed consent to participate in the trial, which was approved by the IRB and the ethics committee of CGMH, in compliance with Taiwanese law (IRB no. 97-1413A3). This trial is also registered with the US ClinicalTrials.gov (NCT01276314).

\section{Author contributions}

CWW participated in the study design, measurement of immunologic effects, and analysis and interpretation of the final data, as well as in the drafting and approval of the final manuscript. LYY, SIH, SCS, and CJC participated in the conception and design of the study. $\mathrm{HCH}$, CHY, CBC, RCYH, YCC, CWL, and JCH were involved in the recruitment of participants from clinics. SWC participated in the measurement and analysis of the immunologic effects results. LYY, WYC, CBC, WLF, and CJC participated in the analysis and interpretation of the clinical results. LYY and CJC helped generate the random number lists. LFH and YYWL helped record the participants' clinical information such as adverse events/serious adverse events data, skin-healing time, and oral mucosa-healing time. WHC was responsible for the recruitment of participants as well as the design and supervision of the study and revision of the manuscript. All authors read and approved the final manuscript. The study was designed and performed by the academic investigators. The authors confirm the accuracy, completeness, and fidelity of all study data and analyses, and all authors read and approved the final version of this manuscript.

\section{Acknowledgments}

We especially thank Chyong-Huey Lai, Director of the Clinical Trial Center at CGMH, in Linkou, Taiwan, for her design and support of the clinical trial. We also thank the staff of the Biostatistical Center for Clinical Research at CGMH (Linkou, Taiwan) and the Whole-Genome Research Core Laboratory of Human Diseases at CGMH (Keelung, Taiwan) for their analytic support. We thank the RegiSCAR committee for the second evaluation of several of the participants diagnosed with SJS-TEN. We also thank the TSCAR Consortium for referring SJS-TEN patients to our study. See the Supplemental Acknowledgments for details on the TSCAR Consortium.

Address correspondence to: Wen-Hung Chung, Department of Dermatology, Drug Hypersensitivity Clinical and Research Center, Chang Gung Memorial Hospital, No. 5, Fusing Street, Taoyuan, 333 Taiwan. Phone: 886.3.3281200 ext. 8495; Email: wenhungchung@yahoo.com or chung1@cgmh.org.tw.
1. Roujeau JC, et al. Medication use and the risk of Stevens-Johnson syndrome or toxic epidermal necrolysis. NEngl JMed.1995;333(24):1600-1607.

2. Halevy S, et al. Allopurinol is the most common cause of Stevens-Johnson syndrome and toxic epidermal necrolysis in Europe and Israel. $\mathrm{JAm}$ Acad Dermatol. 2008;58(1):25-32.

3. Ferrell PB, McLeod HL. Carbamazepine, HLA-B $^{*} 1502$ and risk of Stevens-Johnson syndrome and toxic epidermal necrolysis: US FDA recommendations. Pharmacogenomics. 2008;9(10):1543-1546.

4. Phillips EJ, Chung WH, Mockenhaupt M, Roujeau JC, Mallal SA. Drug hypersensitivity: pharmacogenetics and clinical syndromes. JAllergy Clin Immunol. 2011;127(3 Suppl):S60-S66.

5. Chung WH, Wang CW, Dao RL. Severe cutaneous adverse drug reactions. J Dermatol. 2016;43(7):758-766.

6. Chung WH, et al. Medical genetics: a marker for Stevens-Johnson syndrome. Nature. 2004;428(6982):486.

7. Hung SI, et al. HLA-B`5801 allele as a genetic marker for severe cutaneous adverse reactions caused by allopurinol. Proc Natl Acad Sci US A. 2005;102(11):4134-4139.

8. Chessman D, et al. Human leukocyte antigen class I-restricted activation of CD8+ T cells provides the immunogenetic basis of a systemic drug hypersensitivity. Immunity. 2008;28(6):822-832.

9. Zhang FR, et al. HLA-B ${ }^{*} 13: 01$ and the dapsone hypersensitivity syndrome. $\mathrm{N}$ Engl J Med. 2013;369(17):1620-1628.

10. Ko TM, et al. Shared and restricted T-cell receptor use is crucial for carbamazepine-induced Stevens-Johnson syndrome. J Allergy Clin Immunol. 2011;128(6):1266-1276.e11.

11. Wei CY, Chung WH, Huang HW, Chen YT, Hung SI. Direct interaction between HLA-B and carbamazepine activates $\mathrm{T}$ cells in patients with Stevens-Johnson syndrome. J Allergy Clin Immunol. 2012;129(6):1562-9.e5.

12. Chung WH, et al. Granulysin is a key mediator for disseminated keratinocyte death in StevensJohnson syndrome and toxic epidermal necrolysis. Nat Med. 2008;14(12):1343-1350.

13. Wang CW, et al. A new nucleic acid-based agent inhibits cytotoxic T lymphocyte-mediated immune disorders. J Allergy Clin Immunol. 2013;132(3):713-722.e11.

14. Deng A, Chen S, Li Q, Lyu SC, Clayberger C, Krensky AM. Granulysin, a cytolytic molecule, is also a chemoattractant and proinflammatory activator. J Immunol. 2005;174(9):5243-5248.

15. Tewary P, et al. Granulysin activates antigenpresenting cells through TLR4 and acts as an immune alarmin. Blood. 2010;116(18):3465-3474.

16. Walch $\mathrm{M}$, et al. Cytotoxic cells kill intracellular bacteria through granulysin-mediated delivery of granzymes. Cell. 2014;157(6):1309-1323.

17. Wojtkiewicz A, Wysocki M, Fortuna J, Chrupek M, Matczuk M, Koltan A. Beneficial and rapid effect of infliximab on the course of toxic epidermal necrolysis. Acta Derm Venereol. 2008;88(4):420-421.

18. Posadas SJ, et al. Delayed reactions to drugs show levels of perforin, granzyme B, and Fas-L to be related to disease severity. J Allergy Clin Immunol. 2002;109(1):155-161.

19. Paquet P, Piérard GE. Erythema multiforme and toxic epidermal necrolysis: a comparative study. Am J Dermatopathol.1997;19(2):127-132. 
20. Paul C, et al. Apoptosis as a mechanism of keratinocyte death in toxic epidermal necrolysis. Br J Dermatol. 1996;134(4):710-714.

21. Viard-Leveugle I, et al. TNF- $\boldsymbol{\alpha}$ and IFN- $\gamma$ are potential inducers of Fas-mediated keratinocyte apoptosis through activation of inducible nitric oxide synthase in toxic epidermal necrolysis. J Invest Dermatol. 2013;133(2):489-498.

22. Law EH, Leung M. Corticosteroids in StevensJohnson Syndrome/toxic epidermal necrolysis: current evidence and implications for future research. Ann Pharmacother. 2015;49(3):335-342.

23. Viard I, et al. Inhibition of toxic epidermal necrolysis by blockade of CD95 with human intravenous immunoglobulin. Science. 1998;282(5388):490-493.

24. Reese D, Henning JS, Rockers K, Ladd D, Gilson R. Cyclosporine for SJS/TEN: a case series and review of the literature. Cutis. 2011;87(1):24-29.

25. Schneck J, Fagot JP, Sekula P, Sassolas B, Roujeau JC, Mockenhaupt M. Effects of treatments on the mortality of Stevens-Johnson syndrome and toxic epidermal necrolysis: A retrospective study on patients included in the prospective EuroSCAR Study. J Am Acad Dermatol. 2008;58(1):33-40.

26. Knowles S, Shear NH. Clinical risk management of Stevens-Johnson syndrome/toxic epidermal necrolysis spectrum. Dermatol Ther. 2009;22(5):441-451.

27. Famularo G, Di Dona B, Canzona F, Girardelli CR, Cruciani G. Etanercept for toxic epidermal necrolysis. Ann Pharmacother. 2007;41(6):1083-1084.

28. Gubinelli E, Canzona F, Tonanzi T, Raskovic D, Didona B. Toxic epidermal necrolysis successfully treated with etanercept. J Dermatol. 2009;36(3):150-153.

29. Gunawardane ND, Menon K, Guitart J, Cotliar JA. Purpura fulminans from meningococcemia mimicking Stevens-Johnson syndrome in an adult patient taking etanercept. Arch Dermatol. 2012;148(12):1429-1431.

30. Napolitano M, Giampetruzzi AR, Didona D, Papi M, Didona B. Toxic epidermal necrolysis-like acute cutaneous lupus erythematosus successfully treated with a single dose of etanercept: report of three cases. J Am Acad Dermatol. 2013;69(6):e303-e305.

31. Paradisi A, Abeni D, Bergamo F, Ricci F, Didona D, Didona B. Etanercept therapy for toxic epidermal necrolysis. J Am Acad Dermatol. 2014;71(2):278-283.

32. Didona D, Paolino G, Garcovich S, Caposiena Caro RD, Didona B. Successful use of etanercept in a case of toxic epidermal necrolysis induced by rituximab. JEur Acad Dermatol Venereol. 2016;30(10):e83-e84.

33. Fischer M, Fiedler E, Marsch WC, Wohlrab J. Antitumour necrosis factor-alpha antibodies (infliximab) in the treatment of a patient with toxic epidermal necrolysis. Br J Dermatol. 2002;146(4):707-709.

34. Hunger RE, Hunziker T, Buettiker U, Braathen LR, Yawalkar N. Rapid resolution of toxic epidermal necrolysis with anti-TNF-alpha treatment. JAllergy Clin Immunol. 2005;116(4):923-924.

35. Kreft B, Wohlrab J, Bramsiepe I, Eismann R, Winkler M, Marsch WC. Etoricoxib-induced toxic epidermal necrolysis: successful treatment with infliximab. J Dermatol. 2010;37(10):904-906.

36. Zárate-Correa LC, Carrillo-Gómez DC, RamírezEscobar AF, Serrano-Reyes C. Toxic epidermal necrolysis successfully treated with infliximab. J Investig Allergol Clin Immunol. 2013;23(1):61-63.

37. Scott LJ. Etanercept: a review of its use in autoimmune inflammatory diseases. Drugs. 2014;74(12):1379-1410.

38. Bastuji-Garin S, Fouchard N, Bertocchi M, Roujeau JC, Revuz J, Wolkenstein P. SCORTEN: a severity-of-illness score for toxic epidermal necrolysis. J Invest Dermatol. 2000;115(2):149-153.

39. Wolkenstein P, et al. Randomised comparison of thalidomide versus placebo in toxic epidermal necrolysis. Lancet. 1998;352(9140):1586-1589.

40. Revuz J, et al. Toxic epidermal necrolysis. Clinical findings and prognosis factors in 87 patients. Arch Dermatol. 1987;123(9):1160-1165.

41. Beato M, Chávez S, Truss M. Transcriptional regulation by steroid hormones. Steroids. 1996;61(4):240-251.

42. Wang AS, Armstrong EJ, Armstrong AW. Corticosteroids and wound healing: clinical considerations in the perioperative period. Am J Surg. 2013;206(3):410-417.

43. Goffe B. Etanercept (Enbrel) -- an update. Skin Therapy Lett. 2004;9(10):1-4,-9.

44. Narum S, Westergren T, Klemp M. Corticosteroids and risk of gastrointestinal bleeding: a systematic review and meta-analysis. BMJOpen. 2014;4(5):e004587.

45. Kida Y, Kuwano K, Zhang Y, Arai S. Acholeplasma laidlawii up-regulates granulysin gene expression via transcription factor activator protein- 1 in a human monocytic cell line, THP-1. Immunology. 2001;104(3):324-332.

46. Sakaguchi S, Miyara M, Costantino CM, Hafler DA. FOXP3+ regulatory T cells in the human immune system. Nat Rev Immunol. 2010;10(7):490-500.
47. Azukizawa H, Sano S, Kosaka H, Sumikawa Y, Itami S. Prevention of toxic epidermal necrolysis by regulatory T cells. Eur J Immunol. 2005;35(6):1722-1730.

48. Schrijvers R, Gilissen L, Chiriac AM, Demoly P. Pathogenesis and diagnosis of delayed-type drug hypersensitivity reactions, from bedside to bench and back. Clin Transl Allergy. 2015;5:31.

49. Takahashi R, Kano Y, Yamazaki Y, Kimishima M, Mizukawa Y, Shiohara T. Defective regulatory $\mathrm{T}$ cells in patients with severe drug eruptions: timing of the dysfunction is associated with the pathological phenotype and outcome. Jimmunol. 2009;182(12):8071-8079.

50. Hanafusa T, Azukizawa H, Matsumura S, Katayama I. The predominant drug-specific T-cell population may switch from cytotoxic $\mathrm{T}$ cells to regulatory $\mathrm{T}$ cells during the course of anticonvulsant-induced hypersensitivity. J Dermatol Sci. 2012;65(3):213-219.

51. Ehrenstein MR, et al. Compromised function of regulatory $\mathrm{T}$ cells in rheumatoid arthritis and reversal by anti-TNFalpha therapy. J Exp Med. 2004;200(3):277-285.

52. Valencia X, Stephens G, Goldbach-Mansky R, Wilson M, Shevach EM, Lipsky PE. TNF downmodulates the function of human CD4+CD25hi T-regulatory cells. Blood. 2006;108(1):253-261.

53. Huang Z, et al. Anti-TNF- $\alpha$ therapy improves Treg and suppresses Teff in patients with rheumatoid arthritis. Cell Immunol. 2012;279(1):25-29.

54. Roujeau JC. Clinical heterogeneity of drug hypersensitivity. Toxicology. 2005;209(2):123-129.

55. Auquier-Dunant A, et al. Correlations between clinical patterns and causes of erythema multiforme majus, Stevens-Johnson syndrome, and toxic epidermal necrolysis: results of an international prospective study. Arch Dermatol. 2002;138(8):1019-1024.

56. Naranjo CA, et al. A method for estimating the probability of adverse drug reactions. Clin Pharmacol Ther. 1981;30(2):239-245.

57. Arbache ST, Nogueira TG, Delgado L, Miyamoto $\mathrm{D}$, Aoki V. Immunofluorescence testing in the diagnosis of autoimmune blistering diseases: overview of 10-year experience. An Bras Dermatol. 2014;89(6):885-889.

58. Gerull R, Nelle M, Schaible T. Toxic epidermal necrolysis and Stevens-Johnson syndrome: a review. Crit Care Med. 2011;39(6):1521-1532.

59. Lydersen S, Langaas M, Bakke Ø. The exact unconditional z-pooled test for equality of two binomial probabilities: optimal choice of the Berger and Boos confidence coefficient. J Stat Comput Simul. 2012;82(9):1311-1316. 